\title{
Strong Scattering of High Power Millimeter Waves in Tokamak Plasmas with Tearing Modes
}

\author{
E. Westerhof, ${ }^{1}$ S. K. Nielsen, ${ }^{2}$ J. W. Oosterbeek, ${ }^{3}$ M. Salewski, ${ }^{2}$ M. R. De Baar, ${ }^{1}$ W. A. Bongers, ${ }^{1}$ A. Bürger, ${ }^{3}$ \\ B. A. Hennen, ${ }^{1}$ S. B. Korsholm, ${ }^{2}$ F. Leipold, ${ }^{2}$ D. Moseev, ${ }^{2}$ M. Stejner, ${ }^{2}$ D. J. Thoen, ${ }^{1}$ and the TEXTOR Team \\ ${ }^{1}$ FOM-Institute for Plasma Physics Rijnhuizen, Association EURATOM-FOM Trilateral Euregio Cluster, Nieuwegein, \\ The Netherlands* \\ ${ }^{2}$ Association EURATOM-Ris $\phi$ DTU, Ris $\phi$ National Laboratory for Sustainable Energy, Technical University of Denmark, \\ DK-4000 Roskilde, Denmark \\ ${ }^{3}$ Institute for Energy Research-Plasma Physics, Forschungszentrum Jülich GmbH, Association EURATOM-FZJ, \\ Trilateral Euregio Cluster, 52425 Jülich, Germany \\ (Received 14 April 2009; published 14 September 2009)
}

\begin{abstract}
In tokamak plasmas with a tearing mode, strong scattering of high power millimeter waves, as used for heating and noninductive current drive, is shown to occur. This new wave scattering phenomenon is shown to be related to the passage of the $O$ point of a magnetic island through the high power heating beam. The density determines the detailed phasing of the scattered radiation relative to the $O$-point passage. The scattering power depends strongly nonlinearly on the heating beam power.
\end{abstract}

High power millimeter waves are routinely used for heating and noninductive current generation in tokamak plasmas [1]. Since the exchange of energy between waves and plasma occurs due to resonant interaction of the wave fields with the gyromotion of the electrons, these schemes of heating and current drive are referred to as electron cyclotron resonance heating $(\mathrm{ECRH})$ and electron cyclotron current drive (ECCD), respectively. Well focused wave beams combined with the resonant plasma-wave interaction allow one to deposit power and to drive noninductive current very locally. This makes ECRH and ECCD particularly useful as actuators in control of magnetohydrodynamic instabilities, in particular, neoclassical tearing modes (NTMs) [2]. The control of NTMs is critical to the success of the international ITER experiment, which is to demonstrate the feasibility of fusion energy production [3]. A major task of the ITER ECRH systems will be the control of NTMs [4]. The development of closed loop control of NTMs by ECRH is actively pursued on many tokamaks [5-8]. High power millimeter waves are also used as probe beams for collective Thomson scattering (CTS): the scattering spectrum is used to infer a onedimensional velocity distribution of the ions [9]. Expected CTS signal levels are much smaller than the electron cyclotron emission (ECE) background.

Towards the development of closed loop control of tearing modes on the Tokamak Experiment for Technology Oriented Research (TEXTOR; major radius $R=1.75 \mathrm{~m}$ and minor radius $a=0.46 \mathrm{~m}$ ) [10], a dedicated sensor has been developed: an ECE diagnostic viewing the plasma along the beam line of the ECRH system (inline ECE) [11]. Identical viewing lines for the actuator and the sensor obviate the need for equilibrium reconstruction in order to match a sensor derived location with a desired actuator position. Localization of a structure in the sensor spectrum at the actuator frequency guarantees power deposition on the structure [12]. Of course such a setup also functions as a backscattering diagnostic. However, based on the small scattering cross section for CTS, the backscattered signal was expected to be insignificant. In addition, a CTS diagnostic is available [13-15]. The CTS viewing mirror is located in the same poloidal cross section just above the ECRH launching mirror. Both mirrors can be rotated in both poloidal and toroidal directions. TEXTOR has two gyrotrons sharing the same beam line to the plasma $[16,17]$. The $110 \mathrm{GHz}, 500 \mathrm{~kW}, 200 \mathrm{~ms}$ gyrotron is used exclusively for CTS experiments while the $140 \mathrm{GHz}$, $1 \mathrm{MW}, 10 \mathrm{~s}$ gyrotron is used for ECRH and ECCD. A schematic of the experimental setup is drawn in Fig. 1. In the geometry shown, the CTS scattering region coincides with the (rotating) 2/1 island on the low-field side, which is typical for most data shown in this Letter.

Commissioning of the inline-ECE system led to the chance discovery of a regime of strong scattering: in discharges with a rotating tearing mode, strong spikes in the radiation intensity are seen in phase with the island rotation. These spikes at $138.5 \mathrm{GHz}$ lead to saturation of this channel and compression of all other inline-radiometer channels $(132.5,135.5,141.5,144.5$, and $147.5 \mathrm{GHz})$. Apart from being a nuisance to the application of inline ECE as the main sensor in a control loop, this scattering is an unexpected, interesting new physical phenomenon. To identify its window of occurrence and to guide possible explanations, this new scattering phenomenon has been characterized extensively. A new spectrometer was attached to the inline-ECE antenna through a $3 \mathrm{~dB}$ coupler. It consists of a heterodyne front end with an analog-todigital converter and a compact personal computer interface. It records bursts of the frequency down-converted signal with a sampling rate of $8 \mathrm{GHz}$ resulting in a resolved spectrum with a $4 \mathrm{GHz}$ bandwidth below the $140 \mathrm{GHz}$ mixing frequency [18]. The tunable length of the recorded 


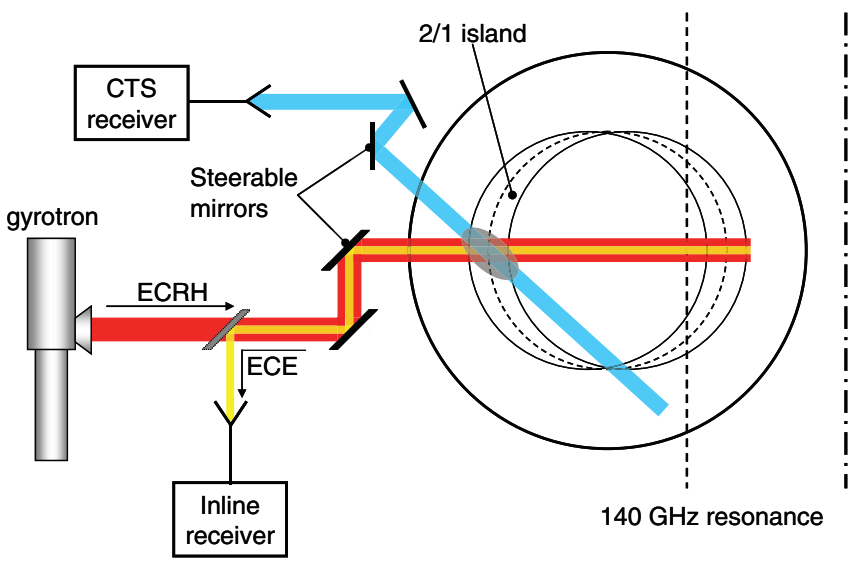

FIG. 1 (color). Schematic of the experimental setup. The gyrotron and both the inline-ECE and CTS diagnostics are viewing the plasma (solid circle) from the low-field side. The gray mirror in the ECRH or inline-ECE beam line indicates the dielectric mirror separating ECE (and backscattered) radiation from the ECRH beam [11]. The gray oval at the intersection of ECRH and CTS beams indicates the CTS scattering region. Backscattered ECRH along the inline-ECE view can come from the entire ECRH beam path in the plasma up to the resonance where the ECRH is absorbed.

bursts determines the frequency resolution (in the range 25-100 MHz) and the maximum rate at which the spectra can be acquired. Inline spectra reported here have been acquired with a frequency resolution of $0.5 \mathrm{MHz}$ and at a rate of $1 \mathrm{kHz}$. The available CTS diagnostic was equipped with a local oscillator at $129.46 \mathrm{GHz}$ in order to set a measurement window of 136-142 GHz. This system has a time resolution of $10 \mu \mathrm{s}$ and a frequency resolution of about $80 \mathrm{MHz}$. The time behavior and spectrum observed by inline ECE and CTS are very similar.

Typical experimental conditions are magnetic field $B_{\phi}=2.25 \mathrm{~T}$, plasma current $I_{p}=300 \mathrm{kA}$, and central line averaged density around $n_{e}(0)=2.5 \times 10^{19} \mathrm{~m}^{-3}$. Resonant magnetic perturbations from the TEXTOR dynamic ergodic divertor (DED), which has been operated in its ac mode at $974 \mathrm{~Hz}$, create a rotating $m=2, n=1$ tearing mode on the $q=2$ surface with minor radius

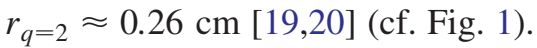

Various overlap scans of the CTS receiver beam with the ECRH beam have been performed; i.e., the receiver antenna is swept such that the associated beam crosses the gyrotron beam. An example of a CTS toroidal viewing angle scan at $R=2 \mathrm{~m}$ is shown in Fig. 2. A strong CTS signal is only observed when the conditions for beam overlap are met. The absence of a significant signal in discharges with blocked CTS transmission line rules out an origin of the signal from spurious gyrotron modes. These results confirm that the signal originates from scattering inside the plasma. Overlap scans at different major radii $(1.6,1.8$, and $2.0 \mathrm{~m})$ at fixed density and magnetic field put the source of the scattered radiation on the lowfield side at a major radius of about $R=2 \mathrm{~m}$. The scattered signal reaches amplitudes up to several $100 \mathrm{keV}$.

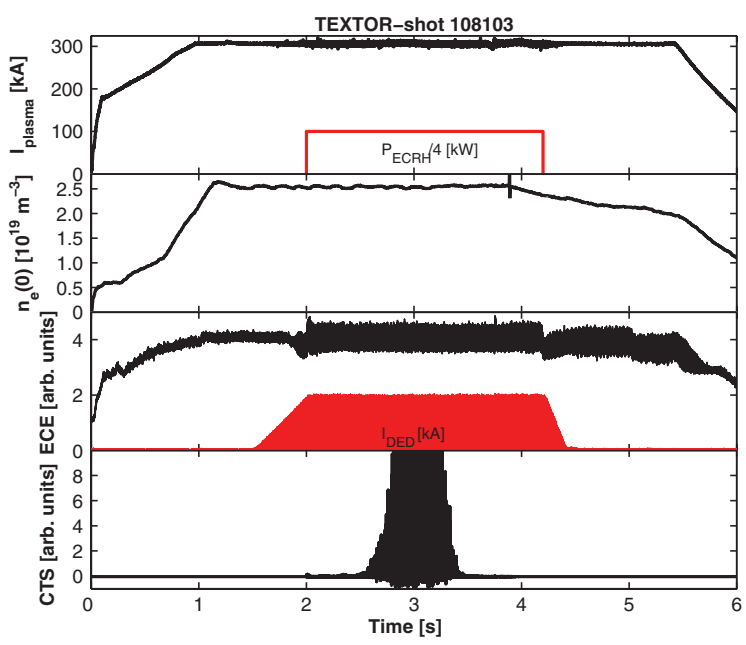

FIG. 2 (color). TEXTOR discharge 108 103. The CTS toroidal viewing angle is scanned during a $400 \mathrm{~kW}$ ECRH pulse. Overlap around $R=2 \mathrm{~m}$ between ECRH (injected with a toroidal angle of $4^{\circ}$ with respect to the inward major radial direction) and CTS occurs near $t=3 \mathrm{~s}$. From top to bottom the frames show the evolution of the plasma current (with ECRH power in an inset), central line averaged electron density, a standard ECE measurement at $141 \mathrm{GHz}$ originating from close to the $q=2$ surface on the high-field side (the insert indicates DED currents), and a CTS signal at $138.58 \mathrm{GHz}$. The oscillations in the $141 \mathrm{GHz}$ ECE signal reveal the $m=2, n=1$ magnetic island triggered by the DED.

Figure 3 shows a zoom of CTS data from the same discharge around $t=3 \mathrm{~s}$, when overlap between ECRH and CTS beams is complete. The currents in the two DED coil sets are also shown. The $m=2, n=1$ magnetic island is locked to the magnetic perturbation field from the DED and thus rotates with the DED frequency of $974 \mathrm{~Hz}$. The times at which the $m=2, n=1$ island $O$ point passes through the ECRH beam on the low-field side are indicated. The scattering is clearly seen to be related to this passage of the $O$ point.

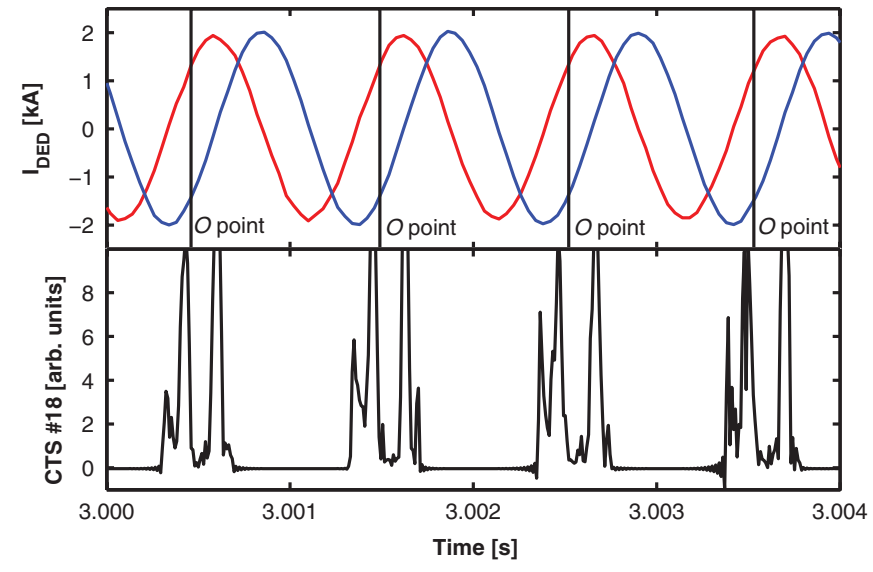

FIG. 3 (color). CTS data from discharge 108103 during beam overlap. The top frame shows the currents in the two sets of DED coils (red for coils 1-4 and blue for coils 5-8). Vertical lines indicate the times that the $O$ point of the island crosses the ECRH beam on the low-field side. 


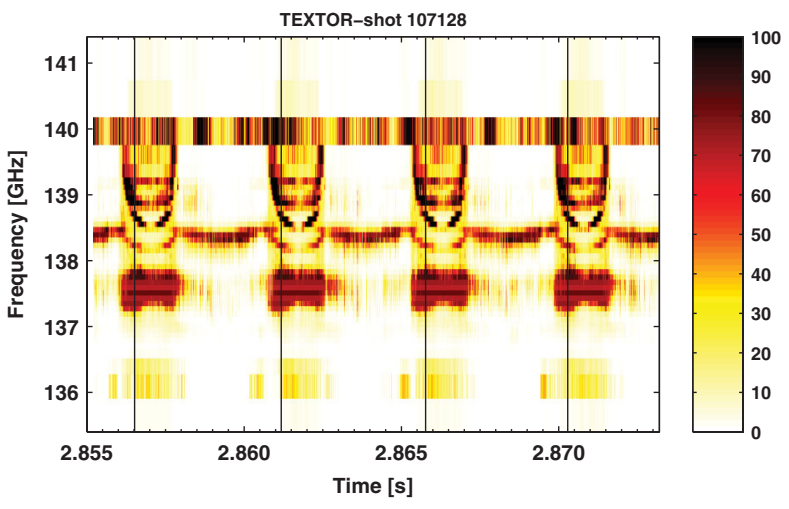

FIG. 4 (color). The CTS spectrum in the presence of a rotating $m=2, n=1$ mode in discharge 107128 . Estimated times of the passage of the island $O$ point through the ECRH beam on the low-field side are indicated by vertical lines.

The scattering shows a rich spectral behavior. This is illustrated for discharge 107128 for almost identical plasma and wave parameters as above, but with a more slowly $(200 \mathrm{~Hz})$ rotating $m=2, n=1$ island so that a better resolution within a rotation period is obtained. Figure 4 shows the CTS spectrum during a period of overlap between the CTS and ECRH beams. The repeating, highly structured features correspond to subsequent island passages. At $137.5 \mathrm{GHz}$, strong signals appear each time in phase with the passage of the $O$ point. In addition, during the onset of this signal three sharp lines chirp down quickly from $140 \mathrm{GHz}$ to $139.3,138.8$, and $138.5 \mathrm{GHz}$, respectively. By the time the strong signal at $137.5 \mathrm{GHz}$ disappears, these lines chirp back again to $140 \mathrm{GHz}$. An additional line is located between 138.2 and $138.5 \mathrm{GHz}$ which is present even in quiescent periods in the channel at 137.5 GHz. This line also exhibits chirping behavior in phase with the island. A weak perturbation is present around $136 \mathrm{GHz}$.

The dependence of the scattering on plasma and wave parameters has been studied extensively. The scattering is observed for toroidal injection angles of the ECRH probe beam ranging from $-20^{\circ}$ to $+20^{\circ}$ with respect to the inward major radial direction. Some variation of the spectral content is seen over this range with only a single, fast down- and up-chirping branch between 140 and $138.8 \mathrm{GHz}$ remaining at the extremes of this range. Variation of the toroidal field from 2.1 to $2.6 \mathrm{~T}$ showed qualitatively little effect on the scattering. In contrast, scans in plasma density revealed a very strong dependence. Figure 5 shows the evolution of the scattering spectrum as observed on the high resolution inline spectrometer in discharge 108115. From $t=2$ to $4 \mathrm{~s}, 600 \mathrm{~kW}$ ECRH is injected. During this time frame, the line average density is ramped from 1.5 to $3.5 \times 10^{19} \mathrm{~m}^{-3}$. Two windows of scattering are identified: one at line average densities below $2 \times 10^{19} \mathrm{~m}^{-3}$ and a second at densities above $2.2 \times 10^{19} \mathrm{~m}^{-3}$ (above $2.8 \times$ $10^{19} \mathrm{~m}^{-3}$, the scattering is again reduced).

The spectra are acquired at a rate of $1 \mathrm{kHz}$ which results in a $-26 \mathrm{~Hz}$ aliasing with the DED frequency and, con-

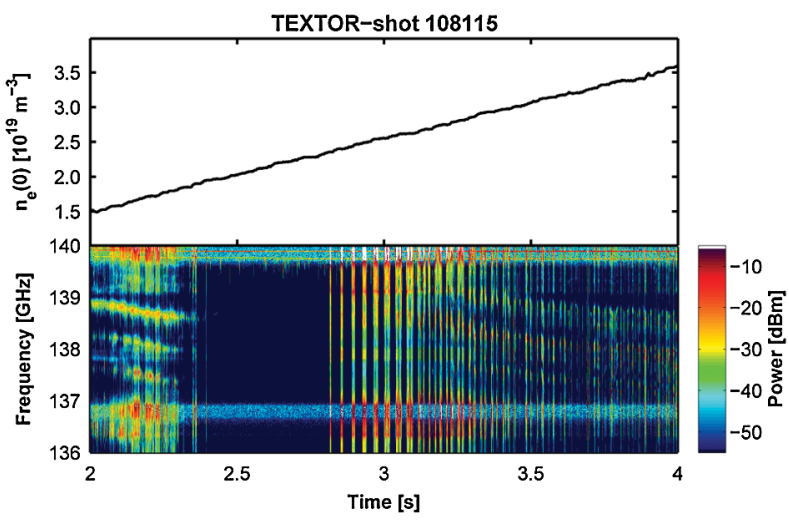

FIG. 5 (color). Inline spectrum of scattered radiation during a density scan in discharge 108115 . A $38 \mathrm{~ms}$ period between scattering pulses is explained by aliasing between the $1 \mathrm{kHz}$ acquisition rate of the inline spectrometer and the $974 \mathrm{~Hz}$ rotation frequency of the magnetic island which is locked to the DED.

sequently, of the magnetic island rotation. This explains the $38 \mathrm{~ms}$ period between pulses of scattered radiation. Figure 6 shows the second period of scattering in more detail from $t=2.8$ to $3.4 \mathrm{~s}$. The currents in the two DED coil sets are also shown. The latter data are subsampled at the same times at which the high resolution inline spectra are recorded. The times at which the low-field side $O$ point crosses the ECRH beam are again indicated. At first, the pulses of scattered radiation exactly coincide with the $O$-point passage. As the density increases, the duration of the scattered pulses increases. At still higher densities, the pulses are split into two parts on opposite sides of the $O$ point. As the density increases further, these two branches move apart and become weaker. Also the frequency of the scattered radiation changes: at the beginning the scattered radiation is peaked near $138.5 \mathrm{GHz}$, while in later phases the peak in the spectrum moves to lower frequencies (down to $138 \mathrm{GHz}$ ).

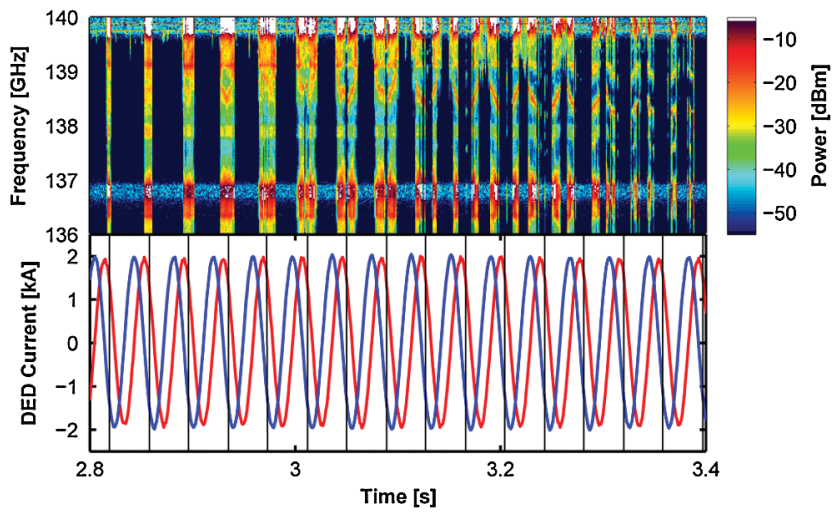

FIG. 6 (color). Inline spectrum of scattered radiation from $t=$ 2.8 to $3.4 \mathrm{~s}$ in discharge 108115 . The bottom frame shows the currents in the DED coil sets (red for coils 1-4 and blue for coils 5-8) subsampled at the times of the recording of the inline spectra. Vertical lines indicate the passage of the $O$ point through the ECRH beam on the low-field side. 


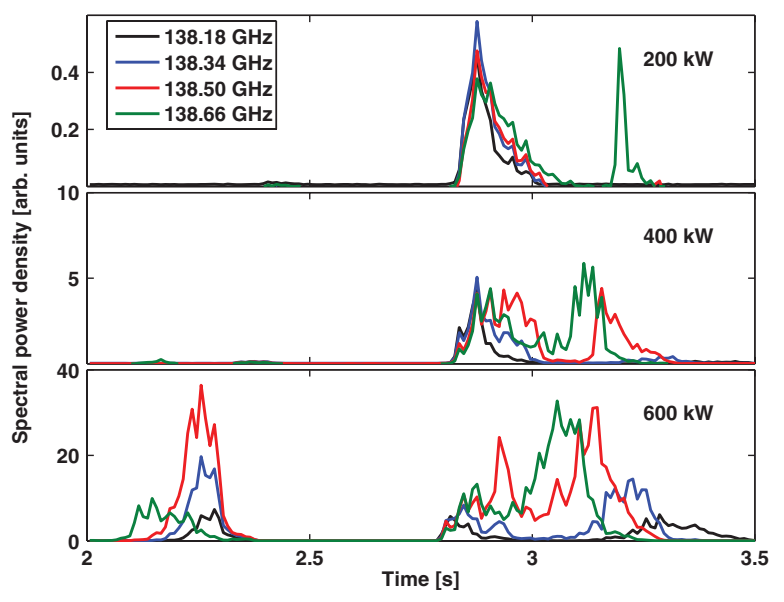

FIG. 7 (color). Evolution of CTS scattering intensity for different ECRH powers. From top to bottom: 200 (discharge 108111 ), 400 (108 114), and $600 \mathrm{~kW}$ (108 115). The data are shown using a $10 \mathrm{~ms}$ sliding time average. CTS channels with frequencies $138.18,138.34,138.50$, and $138.66 \mathrm{GHz}$ are shown. Identical density scans from 1.5 to $3.5 \times 10^{19} \mathrm{~m}^{-3}$ are performed during the $2 \mathrm{~s}$ ECRH pulse (see also Fig. 5).

Discharges with identical density ramps but ECRH powers of 200,400 , and $600 \mathrm{~kW}$ reveal a very strong, nonlinear dependence of the scattering on the injected power. Figure 7 shows the time evolution of the scattering power (using a $10 \mathrm{~ms}$ sliding average) in a number of CTS channels ranging from 138.2 to $138.7 \mathrm{GHz}$. Three periods of scattering can be recognized: the first at about $t=$ $2.1-2.3 \mathrm{~s}$ is only clearly visible at the highest ECRH power, the second at about 2.8-3.0 s is directly followed by the third from 3.0-3.3 s. These correspond to ranges in the central line averaged density of about 1.6 to $1.8 \times$ $10^{19} \mathrm{~m}^{-3}, 2.3$ to $2.5 \times 10^{19} \mathrm{~m}^{-3}$, and 2.5 to $2.8 \times$ $10^{19} \mathrm{~m}^{-3}$, respectively. Figure 7 also illustrates the evolution of the peak frequency in the scattering spectrum. For medium densities, the amplitude of the scattering increases at least as the third power of the injected ECRH power. The scattering at low densities has a threshold-like character and rises more than 3 orders of magnitude when the ECRH power is raised from 400 to $600 \mathrm{~kW}$. In the low density case, the correlation of the scattering with the island phase is less clear.

In conclusion, a new scattering phenomenon of high power millimeter waves as used for ECRH and ECCD in tokamak plasmas has been documented. The scattering occurs only in plasmas with a (rotating) $m=2, n=1$ tearing mode within a well-defined range of densities. In particular, the scattering occurs during passage of the island $O$ point through the ECRH beam on the low-field side of the tokamak. It depends sensitively on the density and shows a strongly nonlinear dependence on the ECRH power. This correlation of wave scattering with a tearing mode has not been foreseen by any existing theory, and no explanation has been found to date. Yet, it is particularly this regime in which ECRH and ECCD find their most prominent application: in the control of NTMs. This makes understanding the phenomenon all the more necessary.

The near absence of scattered radiation at frequencies above the gyrotron frequency is suggestive of an explanation in the form of parametric decay. Parametric decay has been reported from earlier ECRH experiments (see [21] and references therein). However, these observations only relate to high-field side $X$-mode launch of the ECRH. In that case, the waves reach the upper hybrid resonance which favors the occurrence of parametric decay. Strong scattering has also been reported from the $140 \mathrm{GHz}$ CTS experiment on the Wendelstein 7 Advanced Stellarator (W7-AS) [22]. In this case the scattering (observed on both sides of the gyrotron frequency) was only observed in a narrow window of resolved scattered wave vectors close to perpendicular to the magnetic field and was associated with lower hybrid waves generated during neutral beam injection.

This work, supported by the European Communities under the contract of Association between EURATOM and FOM and Ris $\varnothing$ DTU, was carried out within the framework of the European Fusion Programme. The views and opinions expressed herein do not necessarily reflect those of the European Commission. A. Krämer-Flecken and G. W. Spakman are acknowledged for the loan of essential equipment.

*http://www.rijnhuizen.nl

[1] R. Prater, Phys. Plasmas 11, 2349 (2004).

[2] R. J. La Haye, Phys. Plasmas 13, 055501 (2006).

[3] T. C. Hender et al., Nucl. Fusion 47, S128 (2007).

[4] M. A. Henderson et al., Nucl. Fusion 48, 054013 (2008).

[5] A. Isayama et al., Nucl. Fusion 47, 773 (2007).

[6] A. Manini et al., Fusion Eng. Des. 82, 995 (2007).

[7] D. A. Humphreys et al., Phys. Plasmas 13, 056113 (2006).

[8] B. A. Hennen et al., Fusion Eng. Des. 84, 928 (2009).

[9] H. Bindslev, J. Plasma Fusion Res. 76, 878 (2000).

[10] U. Samm, Fusion Sci. Technol. 47, 73 (2005).

[11] J. W. Oosterbeek et al., Rev. Sci. Instrum. 79, 093503 (2008).

[12] E. Westerhof et al., in Proceedings of the 13th Workshop on Electron Cyclotron Emission and Electron Cyclotron Heating, Nizhny Novgorod, Russia, 2004, edited by A. Litvak (IAP/RAS, Nizhny Novgorod, 2005), p. 357.

[13] H. Bindslev et al., Phys. Rev. Lett. 97, 205005 (2006).

[14] S. B. Korsholm et al., Rev. Sci. Instrum. 77, 10 E514 (2006).

[15] S. K. Nielsen et al., Phys. Rev. E 77, 016407 (2008).

[16] E. Westerhof et al., Nucl. Fusion 43, 1371 (2003).

[17] E. Westerhof et al., Fusion Sci. Technol. 47, 108 (2005).

[18] D. J. Thoen et al. (to be published).

[19] H. R. Koslowski et al., Nucl. Fusion 46, L1 (2006).

[20] E. Westerhof et al., Nucl. Fusion 47, 85 (2007).

[21] V. Erckmann, U. Gasparino, Plasma Phys. Controlled Fusion 36, 1869 (1994).

[22] E. V. Suvorov et al., Plasma Phys. Controlled Fusion 37, 1207 (1995). 